\title{
ACTIVE TRAFFIC MANAGEMENT SIGN COMPREHENSION
}

\author{
William A. Perez ${ }^{1} \&$ Brian H. Philips ${ }^{2}$ \\ ${ }^{1}$ SAIC, McLean, Virginia, USA \\ ${ }^{2}$ Federal Highway Administration, McLean, Virginia, USA \\ Email: William.Perez.ctr@dot.gov
}

\begin{abstract}
Summary: Active traffic management (ATM) strategies are being deployed in the United States to deliver additional information to drivers. Per lane variable speed limit and lane control signs are being deployed along with dynamic message signs that display warning and other motorist information. The Manual of Uniformed Traffic Control Devices currently does not provide guidelines or standards for these signs. The present research is the first in a series of studies aimed at providing data that can be used to develop guidelines and standards. This study used laboratory procedures to examine comprehension and preference for various variable speed limit and lane control sign messages. The results indicate that while participants sometimes make errors interpreting some advisory messages, they generally correctly interpreted the lane control and speed limit ATM signs.
\end{abstract}

\section{INTRODUCTION}

Active traffic management (ATM) incorporates a collection of strategies that allows the dynamic management of recurrent and non-recurrent congestion, based on prevailing traffic conditions (Mirshahi et al., 2007). These strategies help to increase peak capacity, smooth traffic flows, and increase safety on busy major highways. Some popular ATM approaches include variable speed limits, lane control signs (also called dynamic lane markings), and hard-shoulder running, controlled by overhead variable message signs.

Although approaches vary, ATM strategies and signing are deployed or in development in the US, the Netherlands, Germany, United Kingdom, Denmark, Australia, Austria, New Zealand, Israel, Greece, and other parts of the world (see Hellinga \& Mandelzys, 2011; Mirshahi et al., 2007). This paper describes research done on two particular types of ATM: variable speed limit (VSL) signing and lane control signing (LCS). Previous research on VSL has shown both safety benefits and efficiency improvements, although the evidence for efficiency improvements has been less conclusive than desired (Papageorgiou, Kosmatopoulos, \& Papamichail, 2008). The safety benefits from VSLs are most often associated with reducing rear-end collisions. This is not surprising since VSLs are effective at reducing speed variability (Lee \& Abdel-Aty, 2008). In contrast, there have been relatively few studies on the impact of LCS on driving behavior, safety, and throughput. However, one study involving a dynamic lane merge traffic control system (LMTCS) that was deployed in Michigan indicated that this type of system can be helpful in reducing aggressive driving, increasing safety, and reducing delay at work zone lane closures (Datta et al., 2004). Other studies have compared the use of graphic displays to their equivalent text messages, and found that graphic displays can improve drivers' abilities to identify available lanes in a problem area and help comprehension for non-native language drivers (e.g., Ullman, Trout, \& Dudek, 2009). 
While there are an increasing number of VSL and LCS deployments in the United States, there is a lack of consistency in the designs. There is also a lack of empirical data on driver behavior as a function of sign design. The study described in this paper examined the design of various LCS and VSL signs and assessed how these designs influenced driver comprehension and behavior. Two current LCS and VSL deployments are underway one in Washington and one Minnesota. The signs used in these deployments were evaluated. The two deployments are using different approaches for sign structure and content (e.g., different symbols for the same type of information). Both sites are operating under a request to experiment with ATM signs. The first series of studies examined how well drivers interpret different configurations, symbols, and messages as a function of scenarios (e.g., one lane closed ahead, congestion ahead). Comprehension was also examined as a function of whether regulatory versus advisory signs were used. In addition, when examining comprehension, participants' signing preferences were also assessed.

\section{METHOD}

The signs were projected on a 60" diagonal LED-LCD display (1080p resolution). Participants sat $5 \mathrm{ft}$. from the display. A computer program was used to present signs and to record participant responses.

Three tests were conducted. First, participant's comprehension of several scenarios was evaluated. A series of signs were presented as they might be encountered on a trip. Figure 1 shows alternative methods of presenting the same information. The picture on the left is based on the Washington deployment and the one on the right on the Minnesota deployment. In Minnesota the ATM signs are being added to existing gantries that already contain guide signs. In the second test participants comprehension of individual LCS and VSL signs was tested. In the third test, preference ratings for individual lane control and variable speed limit signs were obtained.

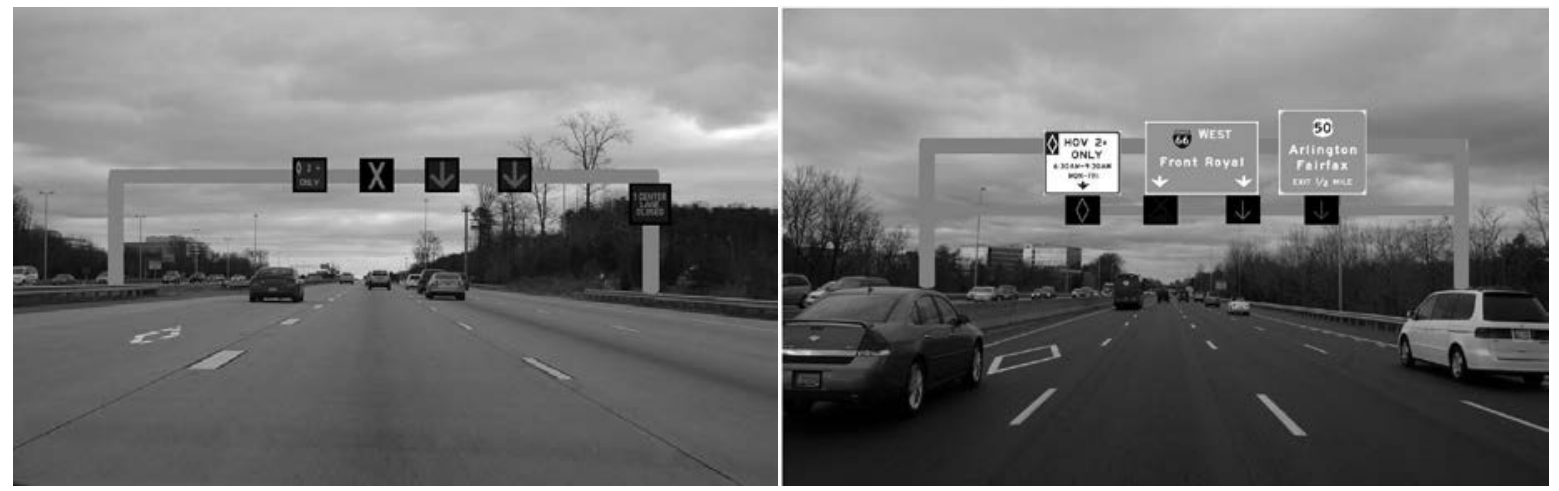

Figure 1. Example slides from the scenario sign comprehension test

\section{Scenario Comprehension}

The participants were presented a series of ATM signs representing a given scenario. There were five ATM sign gantries per scenario. There were five scenarios:

1. Congestion (Recurring Type - i.e., commuter traffic)

2. Incident - 1 Lane Closed (left-center with HOV restricted lane on left)

3. Incident - 2 Right Lanes Closed with Exit Ramp Open 
4. Incident - 1 Single Center Lane Closed (right-center)

5. Resting Condition (normal operations, free flow)

Two variations for the above scenarios were presented to the participants. One variation is similar to the signs used in the Washington deployment and the second is like the deployment in Minnesota. Before the presentation of the pictures in a given scenario the participants were informed of the speed limit, told to assume that they were driving a single occupancy vehicle, and told that they should imagine driving in the second lane from the left. For each of the pictures the following questions were asked: (1) What do the signs mean to you? (2) What action(s) would you engage in based on these signs?

The scenarios were presented for each deployment type (Washington or Minnesota) in a group; that is, all of the scenarios based on a given state's deployment were presented together. With the exception of the "Resting Condition" the order of presentation of four of the scenarios was counterbalanced across participants, as was the order of presentation of deployment types. For half of the participants the "Resting Condition" scenario was presented first and for the others last. Under the "Resting Condition" scenario all of the signs are turned off with the exception of the sign over the HOV lane. This manipulation was included to address a concern that participants may perceive the signs to be out of commission if no content was presented on the signs when they are initially encountered.

\section{Individual Sign Comprehension}

Following the scenario comprehension, the participants were presented individual LCS and VSL signs on an LED-LCD display. Figure 2 presents some of the signs used in this test. Additional variations to the signs in this figure were presented for comprehension testing. The signs were presented in a different random order to each participant. The participants' task was to say what the sign meant.

There were 29 additional signs to those in figure 2 .These variations were generated by changing color, animation, and text in the signs shown in figure 2. In some cases this resulted in signs that were clear violations of MUTCD guidance. For example, this resulted in speed limit signs that was regulatory with respect to the text but in yellow as would be used for advisory speed signs. This manipulation was included to determine if participants can distinguish between regulatory and advisory speed signs in the present context.

\section{Sign Preference}

The participants were shown the LCS and VSL signs in groups (e.g., all merge signs, all speed limit signs) and asked to indicate their preferred sign within each group and the reason for their preference. The signs were presented on the same LCD display used for the previous tests. The participants made verbal responses which an experimenter recorded.

\section{Participants}

There were 26 participants, of which twelve were women. Participants ranged in age from 20 to 56 years old, with a mean age of 36 years. 


\begin{tabular}{|c|c|c|}
\hline Sign Type & Washington & Minnesota \\
\hline $\begin{array}{l}\text { Lane Open -F ully Operational } \\
\text { (green arrows) }\end{array}$ & & \\
\hline $\begin{array}{l}\text { Lane Open - With Caution } \\
\text { (flashing yellow arrow) }\end{array}$ & & \\
\hline $\begin{array}{l}\text { Lane Closed } \\
(\operatorname{red} x)\end{array}$ & & \\
\hline $\begin{array}{l}\text { Lane Closed Ahead } \\
\text { (yellow } \mathrm{x} \text { ) }\end{array}$ & & \\
\hline $\begin{array}{l}\text { Merge } \\
\text { (signs in yellow) }\end{array}$ & & \\
\hline $\begin{array}{l}\text { Variable Speed Signs } \\
\text { (Washington in white and Minnesota in } \\
\text { yellow) }\end{array}$ & & \\
\hline
\end{tabular}

Figure 2. Sample of signs used for comprehension testing

\section{RESULTS}

Most of the analyses conducted below are qualitative in nature; that is, participants' responses to signs are described and evaluated. For the preference rating, a chi-square test of association or ztests for two proportions were used in the interpretation of the results.

\section{Scenario Comprehension}

Minnesota Based Signs. For all the scenarios, participants generally proposed making the appropriate actions based on the sign configurations. The participants tended to misinterpret specific ATM signs as well as the contiguous guide signs. Advisory speed limit signs were frequently interpreted as regulatory speed limit signs. Participants frequently misinterpreted the guide signs where the right lane was thought to be an exit lane.

For the "Resting Condition” scenario the participants generally interpreted the signs correctly and proposed making appropriate actions based on the signs. One half of the participants were shown these signs at the beginning of test and the other half at the end. There appears to be no effect of seeing the Resting Condition sign configurations either first or last.

Washington Based Signs. The Washington based sign configurations included supplementary dynamic message signs (DMS) with traffic condition related messages. These messages were designed to provide drivers a warning about traffic conditions such as slow traffic ahead, closed lanes, and so on. 
With the exception of the "Incident with Two Right Lanes Closed" scenario the participants generally interpreted the signs correctly and proposed making appropriate actions based on the signs. There were very few responses that could be interpreted as incorrect responses. Errors included stating that they would move into the HOV lane because of a closed lane, early lane changes in response to DMS messages, and interpreting a red $\mathrm{X}$ sign as prepare to move out of the lane when in fact the sign meant that the lane was closed.

For the "Incident with Two Right Lanes Closed" scenario the participants had problems interpreting the signs correctly. This scenario included a DMS message indicating that the two right lanes were only open for exit. More than half of the participants interpreted the first sign to mean that the two right lanes were closed ahead. One participant indicated that he was confused because he could not understand how could the right two lanes could be open and closed at the same time.

The results for the "Resting Condition” scenario were similar to those found for the Minnesota based signs.

\section{Individual Sign Testing and Preferences}

Lane Open (Green Arrow). The participants correctly interpreted this sign as indicating that the lane was open and that they could continue and stay in their lane. This was true for the Minnesota and Washington style signs. On the other hand, twenty five of the participants preferred the sign as employed in the Washington deployment $(\mathrm{z}=3.46, p<.001)$. The participants stated that the bolder and larger green arrow was more legible and easier to interpret from far away.

Lane Open with Caution. This sign is supposed to convey the meaning that the lane is open but the driver is to proceed with caution. Only 9 participants had the desired response of proceeding with caution. The participants showed no clear preference for either the flashing or non-flashing yellow arrow $\left(\chi^{2}(1)=1.38, p>.05\right)$. The participants who selected the flashing arrow stated that it was more attention getting. On the other hand, the participants who selected the non-flashing arrow sign stated that the flashing arrow was distracting.

Merge. The results for comprehension were much the same across all of the different signs that were tested. The participants indicated that they were to move out of their lane and merge in the direction indicated by the sign. The participants were asked for their preferences for the merge right/left, merge right, and merge left signs separately. The Minnesota streaming chevrons were selected for merge right or left, e.g., out of current lane, $\left(\chi^{2}(5)=15.08, p<.05\right)$, merge left $\left(\chi^{2}(5)\right.$ $=29.85, p<.001)$, and merge right $\left(\chi^{2}(5)=28.92, p<.001\right)$.

Lane Closed Ahead. The yellow X without text was frequently interpreted as the lane being closed (19 out of 26 responses). However, most participants indicated the correct action required (lane change required). On the other hand, the yellow $\mathrm{X}$ with text was correctly interpreted by all of the participants and they consistently gave the correct response for the action required. The yellow X without text may work well with supplemental DMS that provides information about the road condition (e.g., lane closed ahead). 
For a lane closed ahead without text (yellow X) sign the participants selected the Washington type of $\operatorname{sign}\left(\chi^{2}(1)=7.54, p<.01\right)$. They indicated a preference for a bolder and larger $\mathrm{X}$ that they stated was easier to read. For the yellow X showing a text message (“1 MILE”), no preference was shown for the three signs that were presented $\left(\chi^{2}(1)=1, p>.05\right)$.

Lane Closed. For all three options of this sign, 24 participants provided the correct interpretation. Two of the participants incorrectly stated that the sign meant that the lane was closed ahead. The participants indicated that they were not to use the lane or to exit the lane if they were in it. The participants preferred the red $\mathrm{X}$ as used in the Minnesota deployment (also included the word CLOSED) over the red $X$ as deployed in Washington $(Z=2.13, p<0.02)$. The participants stated the preferred sign was easy to see and the word closed was useful information.

Variable Speed Signs. Seven different variable speed signs were presented using white letters and numbers. Signs showing the words "SPEED LIMIT" along with the number were generally interpreted as speed limit signs. Most of the other signs were also interpreted as speed limit signs. One participant indicated that a sign with the number 45 (no text) meant Route 45, which is a correct response for this sign. In the present context most of the participants interpreted signs with numbers over the lanes as speed limit signs.

Eight different variable speed limit signs with yellow text were presented. These signs were interpreted as being speed limit signs. Twenty four of the participants indicated for all of the different signs that they were to drive 45 miles per hour. For the $45 \mathrm{MPH}$ sign in negative contrast one participant indicated that this was an advisory sign. As in the scenario comprehension testing, advisory speed signs were interpreted as regulatory.

Two regulatory speed limit signs were evaluated with respect to preference. These two signs were the speed limit signs used in Washington either in positive (as used in Washington) or negative (alternative sign) contrast. The positive contrast speed limit $\operatorname{sign}(\mathrm{z}=3.05, p<.01)$ was preferred and the participants stated that positive contrast signs showed better contrast and were easier to read compared to the negative contrast sign.

Four different advisory speed signs were presented to the participants. The positive contrast sign (which is in the MUTCD) was selected by 22 of the participants $\left(\chi^{2}(3)=49.69, p<.001\right.$ ). Participants indicated that this sign showed better contrast than the other advisory speed signs and that it was easier to read. The positive contrast sign used in Minnesota was preferred by 2 participants.

\section{DISCUSSION}

The scenario testing portion of this study employed the signs as deployed in Washington and Minnesota. Participants generally interpreted the sequences of ATM signs correctly. The participants in this study had no previous experience with these types of ATM, which suggests they were for the most part intuitive. Errors included interpreting advisory speed signs as regulatory speed limit signs, incorrectly interpreting the guide signs (not ATM signs), misinterpretation of the yellow X sign without text, and confusion over the meaning of yellow arrows (either flashing or not flashing). Participants were challenged with the two right lanes closed expecting one to be an exit lane. 
Testing of individual LCS and VSL signs for comprehension and preference yielded additional information useful for sign design. Signs such as lane open with caution (yellow arrow), and lane closed ahead (yellow X without text) were not correctly interpreted by most participants. The preference ratings tended to correlate with the comprehension scores. Optimally, one would want to deploy signs that are 1) easy to comprehend and intuitive, and 2) preferred by the drivers. Preference for a given sign was generally related to better perceived legibility, conspicuity, and ease of understanding.

These results are based on static testing where participants need only pay attention to the signs. There was no driving task or other workload present in the test situation. However, ATM signs are deployed in a highway environment where there is traffic and drivers may need to make route choices (e.g., take an exit). Subsequent experiments are planned using a driving simulator to evaluate comprehension as well as the actions that drivers take in response to the signs under a variety of ATM scenarios.

\section{ACKNOWLEDGEMENTS}

Work described in this paper was performed under Contract Number DTFH61-08-C-00006 with the Federal Highway Administration, U.S. Department of Transportation. The authors acknowledge the guidance and support provided by the Office of Operations, MUTCD Team.

\section{REFERENCES}

Datta, T., Schattler, K., Kar, P. and Guha, A. (2004). Development and evaluation of an advanced dynamic lane merge traffic control system for 3 to 2 lane transition areas in work zones. (Technical report). Detroit, MI: Michigan Department of Transportation.

Hellinga, B., Mandelzys, M. (2011). Impact of Driver Compliance on the Safety and Operational Impacts of Freeway Variable Speed Limit Systems, Journal of Transportation Engineering, 137, 260-268.

Lee, C. and Abdel-Aty, M. (2008). Testing effects of warning messages and variable speed limits on driver behavior using driving simulator.” Transportation Research Record, 2069(8), 5564.

Mirshahi, M., Obenberger, J., Fuhs, C.A., Howard, E., Krammes, R.A., Kuhn, B.T., Mayhew, R.M., Moore, M.A., Sahebjam, K., Stone, C.J., and Yung, J.L. (2007). Active TrafficManagement: The Next Step in Congestion Management. (Technical Report, HWAPL-07-012). Washington, DC: Federal Highway Administration.

Papageorgiou, M., Kosmatopoulos, E., and Papamichail, I. (2008). Effects of variable speed limits on motorway traffic flow. Transportation Research Record, 2047(5), 37-48.

Ullman, B., R, Trout, N., D, \& Dudek, C., L. (2009). Use of Graphics and Symbols on Dynamic Message Signs. (Technical Report, Vol. 2009/05). Austin, TX: Texas Department of Transportation. 